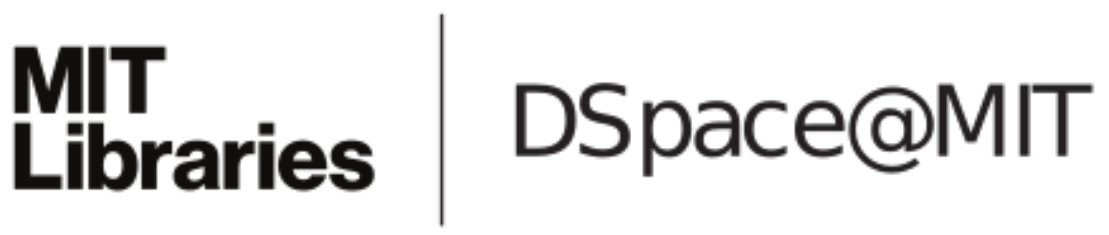

\author{
MIT Open Access Articles
}

\begin{abstract}
Quantitative modeling perspectives on the ErbB system of cell regulatory processes
\end{abstract}

The MIT Faculty has made this article openly available. Please share how this access benefits you. Your story matters.

Citation: Lazzara, Matthew J., and Douglas A. Lauffenburger. "Quantitative modeling perspectives on the ErbB system of cell regulatory processes." Experimental Cell Research 315.4 (2009): 717-725.

As Published: http://dx.doi.org/10.1016/j.yexcr.2008.10.033

Publisher: Elsevier

Persistent URL: http://hdl.handle.net/1721.1/60316

Version: Author's final manuscript: final author's manuscript post peer review, without publisher's formatting or copy editing

Terms of Use: Article is made available in accordance with the publisher's policy and may be subject to US copyright law. Please refer to the publisher's site for terms of use. 


\title{
Quantitative Modeling Perspectives on the ErbB System of Cell Regulatory Processes
}

\author{
Matthew J Lazzara ${ }^{1}$ and Douglas A Lauffenburger ${ }^{2}$ \\ ${ }^{1}$ Department of Chemical and Biomolecular Engineering, \\ University of Pennsylvania, Philadelphia PA 19104 \\ ${ }^{2}$ Departments of Biological Engineering and Biology, \\ Massachusetts Institute of Technology, Cambridge MA 02139
}

V5 19 October 2008 


\begin{abstract}
The complexities of the processes involved in ErbB-mediated regulation of cellular phenotype are broadly appreciated, so much so that it might be reasonably argued that this highly studied system provided significant impetus for the systems perspective on cell signaling processes in general. Recent years have seen major advances in the level of characterization of the ErbB system as well as our ability to make measurements of the system. This new data provides significant new insight, while at the same time creating new challenges for making quantitative statements and predictions with certainty. Here, we discuss recent advances in each of these directions and the interplay between them, with a particular focus on quantitative modeling approaches to interpret data and provide predictive power. Our discussion follows the sequential order of ErbB pathway activation, beginning with considerations of receptor/ligand interactions and dynamics, proceeding to the generation of intracellular signals, and ending with determination of cellular phenotype. As discussed herein, these processes become increasingly difficult to describe or interpret in terms of deterministic models, and we review emerging methodologies to address this complexity.
\end{abstract}




\section{INTRODUCTION}

The constellation of molecular components involved in the regulation of cellular behavior by the ligands and receptors comprising the ErbB family was appreciated to be a highly integrated system comprising complex multi-pathway connections well before the term 'systems biology' was coined. The recently intensified focus on a systems view of cell signaling processes has brought this fundamental aspect of ErbB biology into even sharper relief. Our aim in this article is to focus on the quantitative treatment of this system, with emphasis on efforts coupling computational modeling with experimental measurement toward the objective of gaining insights concerning operation of this system that are not easily ascertained from intuitive observation. We presume that the reader possesses "prior knowledge" from a previous review article of this kind [1], and thus we restrict our discussion largely to publications in the past five years since that publication. Our commentary will be similarly, but not identically, organized. We begin with examination of recent advances in quantitative analysis of the ligand/receptor interaction dynamics, including effects of presentation mode (i.e., autocrine, paracrine, juxtacrine) as well as of receptor trafficking processes. We then move to discuss quantitative analysis of the consequent regulation of cell phenotypic behavior via signaling network activity, first relating ligand/receptor interactions to signals and then relating signals to behavioral responses (see Figure 1).

As a context for the magnitude and interconnectedness of the ErbB system we take the impressive 'comprehensive pathway map' proffered by the Kitano laboratory [2], derived from 242 earlier publications. This map comprises 322 entities involved in 211 interactions among 122 particular protein components. These proteins include the 4 Erb family receptors: ErbB1 (EGFR), ErbB2, ErbB3, and ErbB4; they also include the contemporaneously identified ErbB family ligands: amphiregulin, betacellulin, biregulin, EGF, epiregulin, HB-EGF, neuregulin $1 \mathrm{a} / 1 \mathrm{~b} / 2 \mathrm{a} / 2 \mathrm{~b} / 3 / 4$, and TGF $\alpha$. The additional ligand epigen has also recently been identified [3]. With respect to regulatory network intermediates, downstream of ligand/receptor couplings and upstream of their ultimately regulated processes (transcriptional, metabolic, and cytoskeletal), 32 kinases, 3 ion channels, 22 adaptors, and 6 G-protein subunits were configured into what the authors termed a "bow-tie" structure. By this the authors mean that the ligand/receptor couples 
activate a large number of components in proximal manner, which subsequently converge into a more limited set of pathway intermediates traversing kinase and GTPase nodes, which then influence activities of a larger company of effectors governing metabolic, cytoskeletal, and transcriptional processes that execute cell phenotypic behaviors. This topological conceptualization of ErbB signaling has been emphasized by the Yarden laboratory [4], specifically in relation to the role of this structure in conferring various network control properties. In fact, this view of information funneled through a narrowed signaling network topology between upstream initiating receptorlevel events and downstream execution processes more generally resonates with compatible notion of an identifiable set of canonical pathways - albeit highly complicated in feedbacks and interconnections, of course -- raised in earlier work [5, 6].

In one dimension of systems biology, the goal would be to continually increase the number of identified components and interactions in this network until it is completely characterized. In a complementary dimension, the goal would be to provide quantitative description of as many component levels and interaction rates as possible. An aspiration overarching both dimensions is predictive understanding of the network operation. This would take the form of constructing models capable of predicting what will happen in a yet-unperformed experiment involving the network components, and could be directed toward any of a variety of questions. Our ensuing discussion describes the recent half-decade's accomplishments toward these goals.

\section{LIGAND/RECEPTOR DYNAMICS - BINDING, DIMERIZATION AND TRAFFICKING}

ErbB functionality requires ligand binding, receptor dimerization, and receptor trafficking, and variability in each process may produce differential regulation of the receptors and the downstream signals they control. Such variability is naturally created among diverse cell and tissue types with variable local expression of both ligands and receptors. Distinct ErbB ligands bind ErbB receptors with different affinities, altering the patterns and dynamics of ErbB dimer formation. In addition, the identity of the particular ligand and dimeric receptor formed influences the rate at which receptors traffic.

Because of the complexity and interconnectedness of these processes, quantitative 
computational models are indispensable in the effort to dissect them. We now review recent efforts along these lines.

One convenient avenue for this discussion is the topic of elevated HER2 expression and its impact on ErbB signaling. The well-established involvement of HER2 in breast cancer has led investigators to seek out the mechanisms through which its increased expression drives transformation and metastasis. One classical view holds that there is an inherent predilection of ligand-bound EGFR to interact with HER2 when the latter is present and that the resulting EGFR-HER2 heterodimers are internalization-impaired, resulting in dimers with prolonged signaling. This view is supported by the finding that EGFR-HER2 dimers appear to form more readily than EGFR homodimers when EGF is added to cells expressing both. It is further supported by experiments showing that increasing HER2 expression leads to increased residence times of EGFR at the plasma membrane. Each of these effects would in principle determine the net amounts of EGFR and HER2 present at the cell surface, but the processes are linked. To the extent that an EGFR-HER2 heterodimer does have a reduced capacity for internalization relative to EGFR homodimers, the appearance of these heterodimers in the cell will be automatically biased. To properly uncouple these processes, Hendriks et al. [7] developed a mass-action kinetic model of EGFR-HER2 dimerization and trafficking. By applying this model to results obtained from a set of human mammary epithelial cells engineered to express increasing relative amounts of HER2, Hendriks et al. [7] found that EGFR/EGFR homodimers and EGFR/HER2 heterodimers in fact form with comparable affinities and that EGFR/HER2 heterodimers traffick as individual entities at rates slower than EGFR/EGFR homodimers but faster than HER2 without the presence of EGF. Hendriks et al. [7] further concluded that normal levels of HER2 expression are at the cusp of what is required for efficient heterodimerization with EGFR, reinforcing the notion that regulation of HER2 expression levels may indeed be largely explanatory of differential cell signaling regulation resulting from HER2 over-expression. In a related publication, and working with the same human mammary epithelial cells, Hendricks et al. [8] demonstrated that HER2 over-expression increases the amount of activated EGFR at the plasma membrane by increasing the rate of EGFR recycling and decreasing rate of EGFR 
internalization. While both effects were present, their model revealed that the effect on internalization was most determinative of the increased level of active EGFR at the cell surface. Interestingly, Hendriks et al. [8] were also able to use their model to successfully predict the effects of monoclonal antibody 2C4 (an inhibitor of HER2 dimerization) on EGFR and HER2 levels and compartmental locations. This important finding not only lends credence to the results of such models, but also hints at their potential importance as tools for predicting the efficacy of different molecular therapeutic design approaches.

The interpretation of the effects of expression of other ErbB receptors is subject to similar considerations, and efforts have been made to predict the broader implications of variable ErbB expression profile on dimer formation and trafficking. Shankaran et al. [9] created a deterministic model to predict formation of heterodimers and receptor trafficking based on expression profiles of ErbB1, 2, and 3. Based on independently available parameter estimates, their model predicted that expression of EGFR with HER2 or HER3 results in signaling emanating primarily from the cell surface and impairment of signal downregulation. Moreover, they predicted that simultaneous expression of HER 1-3 favors the formation of HER2-3 heterodimers, thought to be particularly potent mediators of ErbB growth signals. Shankaran et al. [9] further attempted to extend the results of their model to take an important step toward providing predictive capability for cellular outcomes, in this case on the basis of the ErbB dimerization patterns, by employing a phenomenological approach. This methodology bypasses consideration of the actual signals generated and represents one possible approach for predicting actual cellular decision processes. Methodologies for connecting phenotype on the basis of multi-variate signaling data sets are described in detail later.

Variabiliy in ligand/receptor dynamic processes may also occur in and promote disease. The set of somatic mutations in the EGFR locus recently found to correlate with sensitivity to the EGFR kinase inhibitors erlotinib and gefitinib in non-small-cell lung carcinoma provide an especially interesting example [10,11]. The finding that these mutations promote prolonged activation of the EGFR led investigators to probe the trafficking properties of these mutants and find that they were significantly 
internalization-impaired [12]. Investigators further explored the possibility that EGFR internalization impairment might more generally lead increased cellular sensitivity to EGFR inhibition and indeed found that cells expressing WT EGFR but with low rates of EGFR internalization have increased sensitivity to gefitinib relative to WT EGFR cells with normal rates of EGFR internalization. This represents another attempt to predict cellular outcome, in this case response to EGFR inhibition, to upstream events without formal consideration of intracellular signals.

Additional insight on ErbB receptor dynamics has been generated by consideration of apparent underlying design principles. Shankaran et al. [13] developed deterministic models to demonstrate that ErbB receptor downregulation and GPCR desensitization enhance the capacity of these receptors to reliably communicate information to the cell interior. In the context of the ErbB system, Shankaran et al. [13] reached the interesting conclusion that EGFR downregulation yields "low band pass filter" operation, processing inputs only below a certain critical frequency. This feature is predicted to make the system more resilient in its response to external perturbations and improve stability in a resting state. Thus, the endocytic/down-regulatory machinery, traditionally viewed as a method of signal attenuation, may also play a key role in temporal information processing. In a separate study, Shankaran et al. [14] explored the design principles of cellular receptors related to ligand transport. Their parametric analysis revealed fundamental differences in the controlling characteristics of different receptor systems. Whereas transferrin and LDL receptors are avidity controlled (where control depends on ligand capture efficiency) and vitellogenin receptor is consumptioncontrolled (where control depends on internalization efficiency), EGFR is a dual sensitivity receptor. This important distinction leads to the conclusion that EGFR dynamics can efficiently be controlled via modulation of ligand binding properties, ligand availability, and receptor trafficking. As we discuss throughout, evidence indeed exists that modifications of each of these processes may lead to significant alteration of ErbB function.

Significant work remains to be done in the arena of ErbB ligand/receptor dynamics. For example, new information continues to emerge on how distinct ErbB ligands mediate receptor processes. The recently published finding that amphiregulin 
does not promote efficient internalization of EGFR [15], for example, begs the question of whether or not this characterisitic might enable amphiregulin with an ability to drive differential signaling related to its frequent upregulation in response to a variety of cellular stresses. Interestingly, such findings provide new opportunities to rigorously test computational models of ErbB processes as well.

\section{LIGAND/RECEPTOR DYNAMICS - AUTOCRINE/PARACRINE PRESENTATION}

Whereas experiments exploring effects of different ErbB expression profiles or different ErbB ligands are typically undertaken by adding saturating bolus doses of ligand to serum-starved cells, the physiological context is more likely characterized by less dramatic temporal gradients in ligand concentration set by release rates of neighboring cells or a signaling cell itself. Despite these more modest gradients, the analysis of autocrine/paracrine ErbB signaling is not a simple matter, and the complete picture of its complex nature is still evolving. Recent quantitative work elucidating the competing phenomena that determine the extent of autocrine signaling, new biological insights on novel biochemical actors, and evidence of differential phenotypic differences determined by autocrine signaling are now discussed.

In the analysis of autocrine or paracrine signaling, a fundamental question is how the rate of ligand release relates to the amount of receptor activation in a cell. Recent work clarifies that the rates of release do not uniquely determine the magnitude of induced signaling since a released ligand must be recaptured by the releasing cell. Thus, competing effects including molecular diffusive motion and ligand/receptor binding ultimately determine the probability that a released ligand rebinds to a releasing surface, and only the recaptured fraction may contribute to signal. The model put forth by Monine et al. [16] puts these considerations into a quantitative framework in order to compute ligand concentration in autocrine culture as a function of time. A unique feature of their model is direct consideration of the effects of cellular density, a factor not considered in previous models that relied upon single-cell or compartmentalization approximations, via implementation of a boundary homogenization approach. Monine et al. [16] validated the theory developed via comparison with stochastic simulation and 
demonstrated that it could be use to correctly predict ligand accumulation data from human mammary epithelial cells with a tunable autocrine EGF release rate.

New details about autocrine signaling biology continue to emerge as well. Recently, the protein Argos was identified in Drosophila and shown to mitigate EGFmediated autocrine signaling by binding to the Drosophila EGF-homolog Spitz in solution thereby preventing its binding to EGFR [17]. This new finding begs the question of whether or not a mammalian homolog for Argos exists and whether or not proteins with similar function might exist for other ErbB ligands. These findings present new challenges in the analysis of EGFR-mediated cell signaling experiments as well. Indeed, to the extent to which Argos-like proteins exist in solution, they too are subject to the same sorts of reaction-diffusion considerations as EGFR ligands themselves. The balance between these effects would ultimately determine the extent of autocrine signaling. A computational model of these effects was recently put forth by Reeves et al. [18], with results indicating that Argos is involved in modulating Spitz concentration gradients in a way that is explanatory of ectoderm patterning in the wing of Drosophila. The model further demonstrates that the spatial range over which Argos functions may be variable for different functions in different cellular contexts.

Other recent work provides quantitative evidence that the complexities of autocrine versus bolus dose ligand presentation are not lost in determination of cellular phenotype for in vitro experiments. The study by Joslin et al. [19] employed a system of tunable EGF release rate by utilizing fusions of the ligand ectodomain to different membrane anchoring domains that set the rate of proteolytic cleavage of the mature soluble ligand. This system permitted demonstration that increasing autocrine release of EGF in human mammary epithelial cells could drive increasingly efficient cellular migration in a monolayer and this phenotype was directly related to the ability of autocrine signaling to drive more sustained ERK signaling compared to bolus dosing. Interestingly, the authors found that differential ERK activation mediated by autocrine signaling had no impact on cellular proliferation. The findings of this study have obvious important implication for thinking about cellular function in vivo, but they warrant consideration for the design and interpretation of in vitro experiments as well. The finding that the migration phenotype was significantly enhanced in the presence of 
autocrine release potentially suggests that the functions mediated by EGFR might be even more important than previously thought. This study also highlights the care required in attributing an effect to one signaling pathway or another. While increasing autocrine release rate promoted ERK activity and migration, it also resulted in greatly diminished apparent EGFR levels. Thus, on the basis of a protein-based assay alone, one might be mistakenly led to believe that the relative importance of EGFR signaling may be decreasing when in fact the decreased apparent expression level is a reflection of activation and subsequent down-regulation of the receptor.

\section{CUE-TO-SIGNAL DATA AND MODELS}

The first set of publications offering mathematical models representing generation of specific downstream pathway signals due to ErbB receptor/ligand interactions were by Kholodenko et al. [20], Haugh et al. [21], and Schoeberl et al. [22]. Each of these employed mass-action kinetic ordinary differential equation (ODE) models, comprising a relatively small number of molecular species. Kholodenko et al. [20] addressed activation of the Shc/Grb2 and the PLC $\gamma$ pathways during the initial 2 minutes following stimulation with EGF at low and high concentrations; Haugh et al. [21] analyzed PLC $\gamma$-mediated hydrolysis of PIP2 during a 30-minute period following stimulation with either EGF or TGF $\alpha$ across a range of dose concentrations; Schoeberl et al. [22] focused on activation of ERK during a 1-hour period following EGF stimulation at low and high concentrations. All these models restricted their attention to signals generated by EGFR-EGFR homodimers. In each of these efforts, the most notable features were the highly transient nature of downstream pathway activities and the nonlinear relationships between the degree of EGFR activation (characterized by phosphorylation level) and the degree of downstream pathway activation.

Major advances beyond this early body of literature, over the past half-decade, have come from three aspects of expansion of this kind of ODE modeling approach. One aspect is inclusion of other ErbB receptor family members, especially ErbB2 and ErbB3 (relatively little is still known about ErbB4), and EGF family ligands that induce homodimeric and heterodimeric interactions among them. A second aspect is simultaneous incorporation of multiple downstream pathways within a given model. 
Birtwistle et al. [23] demonstrated both of these aspects, building on the original Kholodenko framework by including the Ras/Raf/MEK/ERK and the PI3K/Akt pathways as extensions of the receptor-proximal Shc/Grb2 activation events; the PLC $\gamma$ pathway was discarded, however. Differences were found in ERK and Akt activity dynamics for HRG stimulation relative to EGF stimulation, especially with respect to effects of pharmacological inhibition of the ERK or Akt pathways. While the model could be satisfactorily fit to experimental measurements of ERK and Akt activity dynamics downstream of both EGF and HRG stimulation, it encountered difficulty in predicting the drug inhibition effects. The latter is not surprising, given the complex cross-talk interconnections between kinase pathways - many of which almost certainly remain to be elucidated.

A third aspect of advance has been to more explicitly address the effects of receptor/ligand trafficking processes on downstream signaling dynamics. The importance of this facet has become clear as investigators have broadened their attention to longer time-periods of signaling network activity. Resat et al. [24] developed a novel Monte Carlo stochastic simulation of EGFR homodimer activity and induction of downstream signals, with emphasis on the effects of endocytic internalization and $\mathrm{pH}$ sensitive endosomal ligand/receptor complex dissociation. They spliced the original Kholodenko et al. [20] model for Shc/Grb2 into their receptor/ligand dynamics simulation, and found significant differences for EGF-elicited signaling in comparison to TGF $\alpha$-elicited signaling. Most notably, pathways activated primarily by plasma membrane-associated receptor/ligand complexes were found to be elevated for EGF stimulation relative to TGF $\alpha$ stimulation. These differences arose from the enhanced dissociation of TGF $\alpha / E G F R$ complexes relative to EGF/EGFR complexes, due to the greater $\mathrm{pH}$ sensitivity of the former. Hendriks et al. [25] considered how overexpression of HER2 alters the activity of ERK downstream of EGF stimulation over a 2hour time period, during which the modulation of EGFR-EGFR homodimers and EGFRHER2 heterodimers by endocytic trafficking processes has significant effects. It had been previously observed that ERK signaling is elevated in HER2-overexpressing cells, such as found in many breast cancers, but the mechanism for this elevation was unclear; for instance, speculation had been raised in various quarters that HER2 
possesses an unusually strong capability to activate the ERK pathway. Hendriks et al. [25] used a combination of quantitative experimental measurement and mathematical modeling to ascertain that this was not the case, but rather in fact that EGFR-EGFR homodimers and EGFR-HER2 heterodimers each lead on a per capita basis to essentially identical levels of ERK activity. Moreover, they showed that HER2 heterodimerizes to EGFR with essentially identical association equilibrium constant as EGFR homodimerizes with itself, rather than having an especially high proclivity for interactions with other ErbB members. Finally, they determined that the ratios of ERK activity to EGFR-EGFR homodimer or EGFR-HER2 heterodimer numbers depends on EGF stimulation concentration, with lower ratios for higher EGF concentrations; this result was consistent with the nonlinear, asymptotically saturating dependence of downstream signaling on receptor activity previously found for EGFR-EGFR homodimers.

Substantially ahead of the computational modeling progress on understanding how signals are generated by ErbB receptor/ligand interactions are the experimental measurement advances of the past half-decade. These advances have come from two important technologies in the phosphoproteomic arena: mass spectrometry, and protein microarrays. Major contributions in phosphoproteomic mass spectrometry applications to the ErbB system have been provided by the Mann laboratory $[26,27]$ and the White laboratory [28-30]. Blagoev et al. [26] employed stable isotope labeling of amino acids in cell culture (SILAC) to identify proteins differentially phosphorylated under EGF stimulation relative to control conditions, with selective emphasis on proteins that directly interact with phosphorylated EGFR or phosphorylated Shc by probing cell lysates with an antibody enriching for this category. They identified 28 such proteins whose phosphorylation state was increased by EGF treatment. The later Olsen et al. [27] study also employed SILAC methodology but without the limiting antibody enrichment, and found almost 900 phosphopeptide sites regulated by EGF stimulation. Of these, approximately $94 \%$ were phosphothreonine or phosphoserine sites while only about $6 \%$ were phosphotyrosine sites, due to the vast stoichiometric preponderance of the former classes. The importance of phosphotyrosine sites in the ErbB network is indicated, however, by the observation that this class is more than 3-fold super- 
represented in the EGF-regulated category whereas the phosphothreonine and phosphoserine classes were mildly supra-represented. Zhang et al. [30] used an alternative labeling method, termed ITRAC, to quantitatively distinguish protein phosphosite levels among diverse conditions, and focused on the phosphotyrosine class via selective antibody enrichment. Clustering analysis was applied to elucidate signaling modules sharing similar dynamics, enabling hypotheses concerning participation of previously uncharacterized phosphotyrosine sites in phenotypic cell behavioral processes. Wolf-Yadlin et al. [29] also employed the ITRAC technique, to investigate how the ErbB network is dysregulated by HER2 overexpression under conditions of treatment with either EGF or HRG. Self-organizing map analysis revealed analogous modules whose activity is differentially induced by the various ligand/receptor combinations, such as motility-related signals most strongly elicited in HER2overexpressing cells by EGF treatment. Wolf-Yadlin et al. [28] then extended this methodology to reproducibly capture an even larger set of phosphotyrosine sites, with 208 being quantitatively assessed across 7 time-points following stimulation. Leading contributions on protein microarray applications to this system have come predominantly from the MacBeath laboratory. Jones et al. [31] created a microarray comprising 1272 SH2-binding or PTB-binding domains, and probed them quantitatively with 61 peptides representing phosphotyrosine sites derived from EGFR, HER2, and ErbB3. As with the phosphoproteomic mass spectrometry results, this new technology discovered many novel interactions even at the most proximal locus of activated receptors recruiting docking proteins that serve as connectors to multiple downstream signaling pathways. Kaushansky et al. [32] employed a similar methodology to extend this investigation further to ErbB4, identifying additional previously unrecognized interactions as well as learning that ErbB4 is much narrower in its downstream docking connectors.

In addition to these advances, a final comment should be made on the growing body of knowledge surrounding mechanisms of ErbB signal regulation, especially with regard to signal attenuation. While much of the cue-to-signal literature is focused on steps leading to signal activation, increasing attention is given to the means by which cell signaling networks are negatively regulated after activation. A noteworthy example 
of such efforts is that of Amit et al. [33], who recently identified a module of negative feedback mechanisms in ErbB signaling consisting of a group of genes induced with a time delay after receptor activation. Interference with the transcriptional events leading to the induction of this feedback module generally prolonged EGFR signaling. Perhaps not surprisingly, phosphatases constituted a major element of the induced genes, and their activation was shown to be specific to the downstream pathway activated. This work, and other work discussing newly identified negative and positive feedback controllers of ErbB signaling [34, 35], points to an aspect of ErbB cue-to-signal biology that will undoubtedly receive significant attention moving forward.

Clearly, the scope of experimental data for the ErbB network signaling is increasing rapidly. This encouraging progress at the same time poses a severe challenge to modeling efforts not only in terms of the sheer number of species and interactions but, even more dauntingly, the non-trivial number of components whose associations within network connectivity maps - even those as extensive as that of Oda et al. [2] - are uncertain or even unknown. Not discussed here, due to space limitations, is the growing amount of additional data on ErbB regulation coming from new molecular level insights, for example alterations in receptor activity resulting from small mutations of high relevance to certain ErbB-mediated cancers. These data present new opportunities, and orthogonal ways, to probe ErbB biology, but they bring with them yet more challenges from modeling perspective. New efforts to intelligently reduce the scope of models [36] and deal with the multi-scale nature [37] of this system offer some hope though that the challenges posed by increased experimental data might be tractable.

\section{SIGNAL-TO-RESPONSE DATA AND MODELS}

A most problematic challenge in systems biology, in our view, is predictive understanding of how signals generated by environmental stimuli connect to phenotypic cell behavioral responses. Developing effective models for ligand/receptor interactions and for generation of signals downstream of such interactions is difficult, to be sure, but is fairly straightforward because the biochemistry can in principle be followed experimentally. In contrast, the roads from signals to phenotypic responses are at 
present darkly shrouded, for many reasons. First, governance of phenotype is mediated not only by transcriptional processes but also by metabolic processes and by cytoskeletal processes. While most of post-genomic biology has focused on transcriptional processes and their regulation, how metabolic and cytoskeletal processes are regulated by signaling networks has been relatively understudied. Second, these processes executing phenotypic behaviors involves biophysical mechanisms along with biochemical mechanisms, and the former are less accessible to high-throughput technologies. Third, the regulation of these processes by signaling networks involves the coordinated activity of multiple pathways simultaneously, and perturbation of any pathway (whether by pharmacological agents or by RNAi techniques) almost necessarily leads to concomitant modulation of others, so presuming to understand or predict the effect of a particular pathway perturbation on phenotypic behavioral responses in a uni-variate way is unfortunately naïve.

Not surprisingly, then, there exist to date only few publications successfully describing predictive models for how phenotypic responses are related to signaling network activities. Moreover, these models fall in the more "abstracted" realm, compared to the more "specified" realm, of the computational modeling spectrum [38, 39]. Specified modeling approaches, such as differential equations for the physicochemical mechanisms of biomolecular interactions, require vastly more detailed information than is available for the regulation of transcriptional, metabolic, and cytoskeletal processes, and thus is not a beckoning avenue for the foreseeable future. The more abstracted modeling approaches, such as partial least-squares regression, decision trees, Boolean/fuzzy logic, and Bayesian networks, are much more amenable to extracting relationships among network components - within the signaling pathways themselves and between the signaling pathways and ultimate phenotypic behaviors.

Nagashima et al. [40] measured phosphorylation of EGFR, ERK, and Akt in conjunction with transcriptional profiling for MCF-7 cells stimulated by either EGF or HRG, and applied a multiplicative decomposition computational analysis technique to identify gene sets whose expression is strongly correlated with one or more of these signals. Here, mRNA expression is taken as a surrogate characterization of phenotypic behavior. The key conclusion obtained is that cell differentiation fate is governed most 
strongly by a late wave of signaling, transpiring in the period roughly 50 minutes to 80 minutes post-stimulation, which is more "digital" in nature than the more "graded" early signaling activities, at least for the two downstream pathways (ERK, Akt) examined.

Kumar et al. [41] applied partial least-squares regression modeling to ascertain principal components, representing quantitatively weighted combinations of signals, most strongly correlated with (or against) the phenotypic cell behaviors (proliferation, migration) measured concomitantly with the tyrosine phosphopeptide sites assessed by mass spectrometry in the previously-described study by Wolf-Yadlin et al. [29]. From the 62 phosphosites on 45 proteins quantified across six conditions (two cell types: parental and HER2-overexpressing human mammary epithelial cells; three ligand treatments: EGF, HRG, and autocrine stimulation), this analysis constructed a model comprising two principal components containing 9 phosphosites on 6 key proteins. Training the weighting coefficients on the associated data from the parental cells across the three ligand treatments yielded a model that could successfully predict the behavior of the HER2-overexpressing cells on the basis of the corresponding set of signal measurements. This quantitative combination of 9 phosphosites was proffered to provide a "network gauge", such that its calculation from direct measurement indicates the state of the EGFR/HER2 regulatory system governing proliferation and migration of these human mammary epithelial cells across the landscape of ligand treatment conditions and HER2 expression level. It does not, of course, describe the mechanisms by which the network state is translated into transcriptional, metabolic, and cytoskeletal processes executing the observed proliferation and migration behavior, but instead merely the "information-processing algorithm" characterizing multi-pathway regulatory network operation upstream of those processes. This perspective was tested directly by Kumar et al. [42], who used an analogous partial least-squares modeling approach to predict how modulation of key kinase pathways by pharmacological inhibitors alters migration behavior of HER2-overexpressing mammary epithelial cell migration responses to EGF and HRG. The signal measurements were limited to Western blot assays for phosphorylation state of ERK, Akt, and p38, along with that of EGFR (2 phosphosites), to demonstrate utility for commonly-accessible experimental data-sets. The most important insight gained from this contribution was that whereas a quantitative 
combination of these 5 phosphosites on 4 key proteins could successfully comprehend the effects of kinase inhibitors on cell phenotypic behavior across multiple treatment conditions, no individual signal could by itself. This finding emphasizes that signal-toresponse relationships will in general require multiple signaling pathways to be included in the model, and that attempts to predict how cells will behave on the basis of a single pathway is most likely be in vain; previous manifestations of this crucial notion have been demonstrated in other systems (e.g., TNF $\alpha$ [43, 44], T-cell receptor [45]), and the ErbB system is no different.

A different modeling approach toward elucidating signal-response relationships, that of decision trees, has been applied to EGF-induced migration of fibroblast cells across a range of fibronectin concentrations [46, 47]. The experimental data-set in this study consisted of Western blot measurements of phosphorylation levels of EGFR, $\mathrm{PLC} \gamma, \mathrm{ERK}, \mathrm{PKC} \delta$, and MLC - the latter four residing in pathways regulating various aspects of cell motility biophysics. The decision tree constructed was able to correctly predict the biphasic dose-dependent effect of pharmacological inhibition of $\mathrm{PKC} \delta$; at high concentrations the inhibitor strongly suppressed migration, while at low concentrations it led to enhanced migration.

We note that these signal-response model predictions might be deemed less than completely satisfactory because they require knowledge of signaling activities in multiple pathways, such that predictions of drug effects or gene mutation effects require explicit measurement of the consequent signal modulations for maximal confidence. This situation, however, must remain the case until models for the generation of signals from ligand/receptor interactions are available across a sufficient breadth of pathways and conditions that the signal modulations can themselves be predicted rather than measured.

A final aspect of this topic to note is an increasing interest in connecting cell-level models for dependence of phenotypic functions on molecular properties to models describing higher-level tissue physiology or pathology - producing so-called "multiscale" models. For the ErbB system, this kind of endeavor has been pursued in two main application areas: tumor pathophysiology, and developmental biology. The Shvartsman laboratory has focused on ErbB family ligand diffusion and reaction 
kinetics, along with consequent receptor trafficking and signaling processes, in the context of cellular pattern formation in fly oogenesis $[18,48]$. An especially fascinating feature of this system is the intricate set of feedforward and feedback loops involved in the ErbB ligand/receptor regulatory network, including signaling pathways as well as synthetic and degradative processes. These authors consider the multi-cellular tissue to be an essentially continuous field for molecular processes, permitting description in terms of spatio-temporal differential equations without requiring discrete cells as explicit entities. The Deisboeck laboratory, on the other hand, has used an "agent-based" framework in which individual cells exhibit particular behaviors, to model tumor cell proliferation and migration in glioma [49] and non-small cell lung cancer [50]. Although only a limited set of signaling pathways were incorporated in all these models, their explicit presence in governing phenotypic behaviors of multi-cellular populations within a tissue environment represents an important advance.

\section{SUMMARY AND CONCLUDING REMARKS}

Perhaps not surprisingly, our current level of understanding of ErbB regulatory processes appears most complete for the most receptor-proximal events. Details surrounding the dynamics of receptor/ligand dynamics are well-characterized and involve a relatively small number of molecular interactors. In contrast, the connection between the signals generated by ErbB receptors and the cellular processes they control is much less well-defined. Whereas quantitative deterministic models can be implemented with some confidence at the most receptor-proximal levels, we currently depend mostly upon data-driven approaches to describe more downstream connections to cellular phenotype. It is tempting to predict that eventually the ErbB system will be sufficiently characterized that deterministic methods will be fully applicable to describe ErbB-mediated events, but achievement of that goal remains distant. It is furthermore unclear that such a task is necessary to undertake as data-driven approaches show promise in their predictive ability. Thus, it is possible that these two modeling approaches will essentially meet in the middle at a point where each has been informed by the other and further refinement of the deterministic details is either unnecessary or simply not possible. 


\section{ACKNOWLEDGEMENTS}

This work was partially supported by NCI Integrative Cancer Biology Program grant U54-CA112967 and NCl grant R01-CA096504. 


\section{REFERENCES}

[1] H.S. Wiley, S.Y. Shvartsman, D.A. Lauffenburger, Computational modeling of the EGF-receptor system: a paradigm for systems biology, Trends Cell Biol 13 (2003) 43-50.

[2] K. Oda, Y. Matsuoka, A. Funahashi, H. Kitano, A comprehensive pathway map of epidermal growth factor receptor signaling, Mol Syst Biol 1 (2005) 20050010.

[3] L. Strachan, J.G. Murison, R.L. Prestidge, M.A. Sleeman, J.D. Watson, K.D. Kumble, Cloning and biological activity of epigen, a novel member of the epidermal growth factor superfamily, J Biol Chem 276 (2001) 18265-18271.

[4] A. Citri, Y. Yarden, EGF-ERBB signalling: towards the systems level, Nat Rev Mol Cell Biol 7 (2006) 505-516.

[5] J. Gerhart, 1998 Warkany lecture: signaling pathways in development, Teratology 60 (1999) 226-239.

[6] E. Lee, A. Salic, R. Kruger, R. Heinrich, M.W. Kirschner, The roles of APC and Axin derived from experimental and theoretical analysis of the Wnt pathway, PLoS Biol 1 (2003) E10.

[7] B.S. Hendriks, L.K. Opresko, H.S. Wiley, D. Lauffenburger, Coregulation of epidermal growth factor receptor/human epidermal growth factor receptor 2 (HER2) levels and locations: quantitative analysis of HER2 overexpression effects, Cancer Res 63 (2003) 1130-1137.

[8] B.S. Hendriks, L.K. Opresko, H.S. Wiley, D. Lauffenburger, Quantitative analysis of HER2-mediated effects on HER2 and epidermal growth factor receptor endocytosis: distribution of homo- and heterodimers depends on relative HER2 levels, J Biol Chem 278 (2003) 23343-23351.

[9] H. Shankaran, H.S. Wiley, H. Resat, Modeling the effects of HER/ErbB1-3 coexpression on receptor dimerization and biological response, Biophys $\mathrm{J} 90$ (2006) 3993-4009.

[10] T.J. Lynch, D.W. Bell, R. Sordella, S. Gurubhagavatula, R.A. Okimoto, B.W. Brannigan, P.L. Harris, S.M. Haserlat, J.G. Supko, F.G. Haluska, D.N. Louis, D.C. Christiani, J. Settleman, D.A. Haber, Activating mutations in the epidermal growth factor receptor underlying responsiveness of non-small-cell lung cancer to gefitinib, N Engl J Med 350 (2004) 2129-2139.

[11] R. Sordella, D.W. Bell, D.A. Haber, J. Settleman, Gefitinib-sensitizing EGFR mutations in lung cancer activate anti-apoptotic pathways, Science 305 (2004) 1163-1167.

[12] B.S. Hendriks, G.J. Griffiths, R. Benson, D. Kenyon, M. Lazzara, J. Swinton, S. Beck, M. Hickinson, J.M. Beusmans, D. Lauffenburger, D. de Graaf, Decreased internalisation of erbB1 mutants in lung cancer is linked with a mechanism conferring sensitivity to gefitinib, Syst Biol (Stevenage) 153 (2006) 457-466.

[13] H. Shankaran, H.S. Wiley, H. Resat, Receptor downregulation and desensitization enhance the information processing ability of signalling receptors, BMC Syst Biol 1 (2007) 48.

[14] H. Shankaran, H. Resat, H.S. Wiley, Cell surface receptors for signal transduction and ligand transport: a design principles study, PLoS Comput Biol 3 (2007) e101. 
[15] K.A. Stern, T.L. Place, N.L. Lill, EGF and amphiregulin differentially regulate Cbl recruitment to endosomes and EGF receptor fate, Biochem J 410 (2008) 585594.

[16] M.I. Monine, A.M. Berezhkovskii, E.J. Joslin, H.S. Wiley, D.A. Lauffenburger, S.Y. Shvartsman, Ligand accumulation in autocrine cell cultures, Biophys $\mathrm{J} 88$ (2005) 2384-2390.

[17] D.E. Klein, V.M. Nappi, G.T. Reeves, S.Y. Shvartsman, M.A. Lemmon, Argos inhibits epidermal growth factor receptor signalling by ligand sequestration, Nature 430 (2004) 1040-1044.

[18] G.T. Reeves, R. Kalifa, D.E. Klein, M.A. Lemmon, S.Y. Shvartsman, Computational analysis of EGFR inhibition by Argos, Dev Biol 284 (2005) 523535.

[19] E.J. Joslin, L.K. Opresko, A. Wells, H.S. Wiley, D.A. Lauffenburger, EGFreceptor-mediated mammary epithelial cell migration is driven by sustained ERK signaling from autocrine stimulation, J Cell Sci 120 (2007) 3688-3699.

[20] B.N. Kholodenko, O.V. Demin, G. Moehren, J.B. Hoek, Quantification of short term signaling by the epidermal growth factor receptor, J Biol Chem 274 (1999) 30169-30181.

[21] J.M. Haugh, A. Wells, D.A. Lauffenburger, Mathematical modeling of epidermal growth factor receptor signaling through the phospholipase $C$ pathway: mechanistic insights and predictions for molecular interventions, Biotechnol Bioeng 70 (2000) 225-238.

[22] B. Schoeberl, C. Eichler-Jonsson, E.D. Gilles, G. Muller, Computational modeling of the dynamics of the MAP kinase cascade activated by surface and internalized EGF receptors, Nat Biotechnol 20 (2002) 370-375.

[23] M.R. Birtwistle, M. Hatakeyama, N. Yumoto, B.A. Ogunnaike, J.B. Hoek, B.N. Kholodenko, Ligand-dependent responses of the ErbB signaling network: experimental and modeling analyses, Mol Syst Biol 3 (2007) 144.

[24] H. Resat, J.A. Ewald, D.A. Dixon, H.S. Wiley, An integrated model of epidermal growth factor receptor trafficking and signal transduction, Biophys J 85 (2003) 730-743.

[25] B.S. Hendriks, G. Orr, A. Wells, H.S. Wiley, D.A. Lauffenburger, Parsing ERK activation reveals quantitatively equivalent contributions from epidermal growth factor receptor and HER2 in human mammary epithelial cells, J Biol Chem 280 (2005) 6157-6169.

[26] B. Blagoev, I. Kratchmarova, S.E. Ong, M. Nielsen, L.J. Foster, M. Mann, A proteomics strategy to elucidate functional protein-protein interactions applied to EGF signaling, Nat Biotechnol 21 (2003) 315-318.

[27] J.V. Olsen, B. Blagoev, F. Gnad, B. Macek, C. Kumar, P. Mortensen, M. Mann, Global, in vivo, and site-specific phosphorylation dynamics in signaling networks, Cell 127 (2006) 635-648.

[28] A. Wolf-Yadlin, S. Hautaniemi, D.A. Lauffenburger, F.M. White, Multiple reaction monitoring for robust quantitative proteomic analysis of cellular signaling networks, Proc Natl Acad Sci U S A 104 (2007) 5860-5865.

[29] A. Wolf-Yadlin, N. Kumar, Y. Zhang, S. Hautaniemi, M. Zaman, H.D. Kim, V. Grantcharova, D.A. Lauffenburger, F.M. White, Effects of HER2 overexpression 
on cell signaling networks governing proliferation and migration, Mol Syst Biol 2 (2006) 54.

[30] Y. Zhang, A. Wolf-Yadlin, P.L. Ross, D.J. Pappin, J. Rush, D.A. Lauffenburger, F.M. White, Time-resolved mass spectrometry of tyrosine phosphorylation sites in the epidermal growth factor receptor signaling network reveals dynamic modules, Mol Cell Proteomics 4 (2005) 1240-1250.

[31] R.B. Jones, A. Gordus, J.A. Krall, G. MacBeath, A quantitative protein interaction network for the ErbB receptors using protein microarrays, Nature 439 (2006) 168-174.

[32] A. Kaushansky, A. Gordus, B.A. Budnik, W.S. Lane, J. Rush, G. Macbeath, System-wide Investigation of ErbB4 Reveals 19 Sites of Tyr Phosphorylation that Are Unusually Selective in Their Recruitment Properties, Chem Biol 15 (2008) 808-817.

[33] I. Amit, A. Citri, T. Shay, Y. Lu, M. Katz, F. Zhang, G. Tarcic, D. Siwak, J. Lahad, J. Jacob-Hirsch, N. Amariglio, N. Vaisman, E. Segal, G. Rechavi, U. Alon, G.B. Mills, E. Domany, Y. Yarden, A module of negative feedback regulators defines growth factor signaling, Nat Genet 39 (2007) 503-512.

[34] E. Kario, M.D. Marmor, K. Adamsky, A. Citri, I. Amit, N. Amariglio, G. Rechavi, Y. Yarden, Suppressors of cytokine signaling 4 and 5 regulate epidermal growth factor receptor signaling, J Biol Chem 280 (2005) 7038-7048.

[35] C. Rubin, V. Litvak, H. Medvedovsky, Y. Zwang, S. Lev, Y. Yarden, Sprouty finetunes EGF signaling through interlinked positive and negative feedback loops, Curr Biol 13 (2003) 297-307.

[36] H. Conzelmann, J. Saez-Rodriguez, T. Sauter, E. Bullinger, F. Allgower, E.D. Gilles, Reduction of mathematical models of signal transduction networks: simulation-based approach applied to EGF receptor signalling, Syst Biol (Stevenage) 1 (2004) 159-169.

[37] Y. Liu, J. Purvis, A. Shih, J. Weinstein, N. Agrawal, R. Radhakrishnan, A multiscale computational approach to dissect early events in the Erb family receptor mediated activation, differential signaling, and relevance to oncogenic transformations, Ann Biomed Eng 35 (2007) 1012-1025.

[38] T. Ideker, L.R. Winslow, D.A. Lauffenburger, Bioengineering and systems biology, Ann Biomed Eng 34 (2006) 1226-1233.

[39] K.A. Janes, D.A. Lauffenburger, A biological approach to computational models of proteomic networks, Curr Opin Chem Biol 10 (2006) 73-80.

[40] T. Nagashima, H. Shimodaira, K. Ide, T. Nakakuki, Y. Tani, K. Takahashi, N. Yumoto, M. Hatakeyama, Quantitative transcriptional control of ErbB receptor signaling undergoes graded to biphasic response for cell differentiation, J Biol Chem 282 (2007) 4045-4056.

[41] N. Kumar, A. Wolf-Yadlin, F.M. White, D.A. Lauffenburger, Modeling HER2 effects on cell behavior from mass spectrometry phosphotyrosine data, PLoS Comput Biol 3 (2007) e4.

[42] N. Kumar, R. Afeyan, H.D. Kim, D.A. Lauffenburger, Multipathway model enables prediction of kinase inhibitor cross-talk effects on migration of Her2-

overexpressing mammary epithelial cells, Mol Pharmacol 73 (2008) 1668-1678. 
[43] K.A. Janes, J.G. Albeck, S. Gaudet, P.K. Sorger, D.A. Lauffenburger, M.B. Yaffe, A systems model of signaling identifies a molecular basis set for cytokineinduced apoptosis, Science 310 (2005) 1646-1653.

[44] K. Miller-Jensen, K.A. Janes, J.S. Brugge, D.A. Lauffenburger, Common effector processing mediates cell-specific responses to stimuli, Nature 448 (2007) 604608.

[45] M.L. Kemp, L. Wille, C.L. Lewis, L.B. Nicholson, D.A. Lauffenburger, Quantitative network signal combinations downstream of TCR activation can predict IL-2 production response, J Immunol 178 (2007) 4984-4992.

[46] S. Hautaniemi, S. Kharait, A. Iwabu, A. Wells, D.A. Lauffenburger, Modeling of signal-response cascades using decision tree analysis, Bioinformatics 21 (2005) 2027-2035.

[47] S. Kharait, S. Hautaniemi, S. Wu, A. Iwabu, D.A. Lauffenburger, A. Wells, Decision tree modeling predicts effects of inhibiting contractility signaling on cell motility, BMC Syst Biol 1 (2007) 9.

[48] N. Yakoby, J. Lembong, T. Schupbach, S.Y. Shvartsman, Drosophila eggshell is patterned by sequential action of feedforward and feedback loops, Development 135 (2008) 343-351.

[49] C.A. Athale, T.S. Deisboeck, The effects of EGF-receptor density on multiscale tumor growth patterns, J Theor Biol 238 (2006) 771-779.

[50] Z. Wang, L. Zhang, J. Sagotsky, T.S. Deisboeck, Simulating non-small cell lung cancer with a multiscale agent-based model, Theor Biol Med Model 4 (2007) 50. 


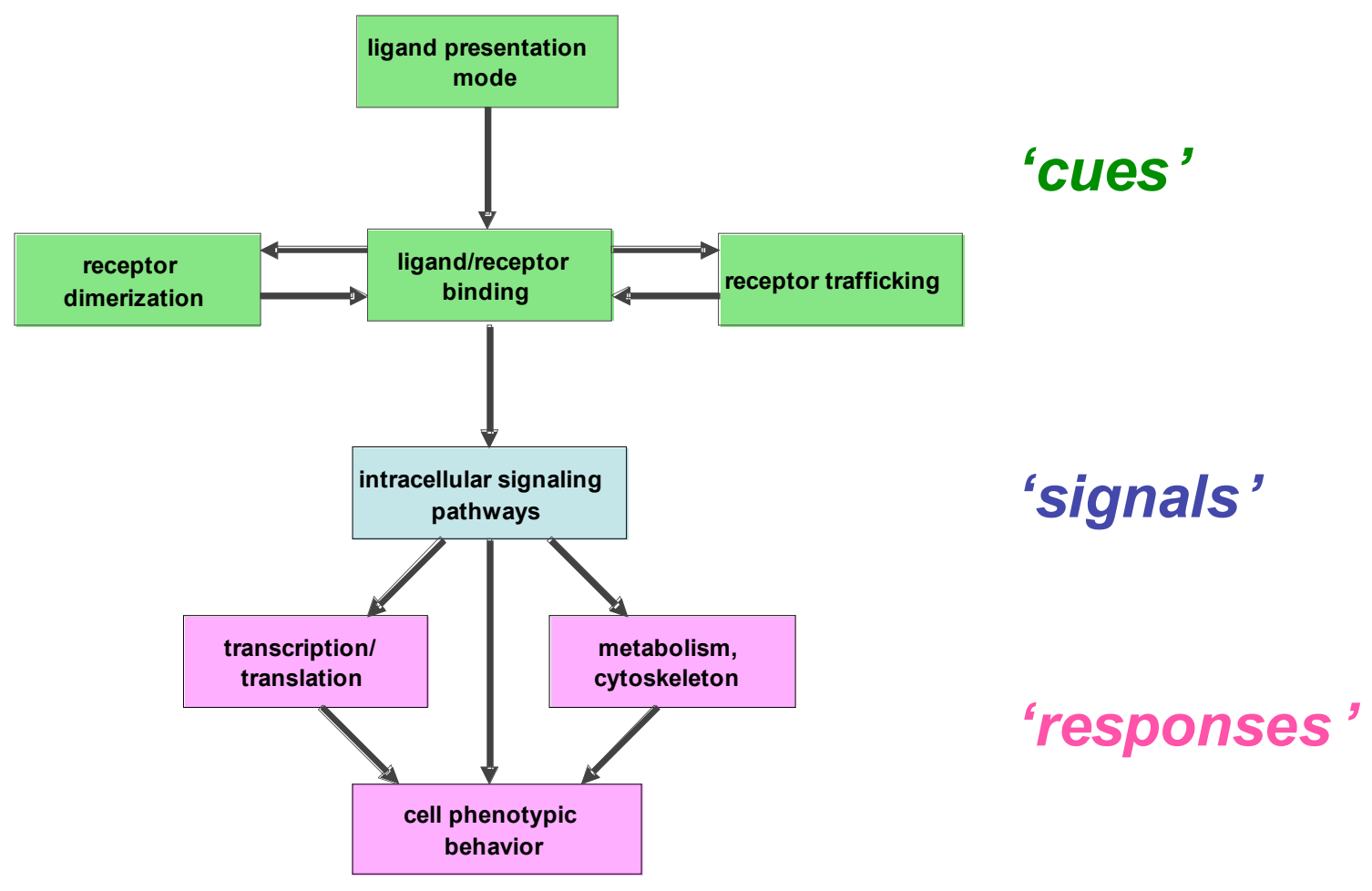

Figure 1. Architecture of the interconnected processes leading to determination of cellular behavior by ErbB receptors. ErbB cue strength and dynamics are set by the mode of ligand presentation, dimerization patterns, receptor trafficking, and avidity of ligand/receptor binding. Ligand-bound receptors activate intracellular signaling pathways, leading to modulation of cellular responses including regulation of transcription/translation, metabolism, cytoskeletal processes, and other measurable phenotypic behaviors. 